\title{
A Conic Sector-Based Methodology for Nonlinear Control Design
}

\author{
Francis J. Doyle III and Manfred Morari* \\ Chemical Engineering 210-41 \\ California Institute of Technology \\ Pasadena CA 91125
}

\begin{abstract}
A design method in presented for the analysis and synthesis of ro bust nonlinear controllers for chemical engineering systems. The method rigorously treats the effect of unmeanured disturbances and unmodeled dynamics on the stability and performance properties of a nonlinear system. The results utilize new extensions of structured singular value theory for analysis and recent synthesis results for approximate linearisation.
\end{abstract}

\section{Introduction}

There has been considerable interest in the application of difierential geometry to the control of nonkinear procese systems over the past five years. The inherently nonlinear nature of chemical process dynamics and the pathological behavior which ean evolve from these aystems has motivated the application of these techniques to process control. Two of the more popular approaches, state linearization and input-output linearization, have received considerable attention. With a few notable exceptions [1], the issues of unmeasured disturbances and unmodeled dynamics in these frameworks have not been property emphasized. In this paper we propose a general methodology to address these iseues.

The analysis toobs employed in this research are extensions of the struetured singular value (SSV) to nonlinear systems [3],[10]. Utilizing the conic sector approximation of a nonlinear operator, it is possible to represent a nonlinear aystem a a nominal LTI plant perturbed by a bounded nonlinear operator. The SSV resulte for nonlinear perturbations are only sufficient and thus conservative. Therefore, the primary objective in this design scheme is the minimization of the sire of the nonlinear perturbations. This is accomplished by two means. First, the nonlinear nature of the plant is minimized by means of approximate linearization vis state transformation and feedback [9]. Second, a tight characterisation of the resultant nonlinearity is achieved with an optimization program. Thus, we arrive at a nearly "linear" plant description which can then be handled with standard robust linear control theory.

The usual Euclidean norm or 2-norm will be used to calculate the norm of vectors in $C^{n}$ or $R^{n}$. For vector signals $e(t)$ this norm is defined to be: $\|e(t)\|^{2}=\int_{-\infty}^{\infty} e^{T}(t) e(t) d t$. The operator norm induced by the 2-norm is:

$$
\sup _{\substack{i \neq 0 \\ \epsilon c_{2}}} \frac{\|G v\|}{\|v\|}=\sup \bar{\sigma}(G(j \omega)) \triangleq\|G\|_{\infty}
$$

where $\mathcal{L}_{2}$ is the apace of functions with bounded 2-norm. This is also the operator norm induced by the power norm defined to be: $\|e(t)\|_{P}^{2}=\lim _{\tau \rightarrow \infty} \int_{-\tau}^{T} \frac{1}{2 T} e^{T}(t) e(t) d t$. The Frobenius norm for matrices in $C^{n \times n}$ is given by $\|A\|_{F}=\left[\sum_{i} \sum_{j}\left|a_{i j}\right|^{2}\right]^{\frac{1}{2}}$. The superscript notation, $f^{(p)}(x)$, will be used to represent a polynomial function of order $\rho$ in the argument.

\section{Feedback Linearization}

The general problem focuses on controtlinear systems of the form:

$$
\dot{x}=f(x)+g(x) u+d(x, t)
$$

\footnotetext{
"Author to whow correspondence hould be adtreand: mime.coltecheda, (818)356-4186
}

where $x \in \mathfrak{X}^{n}, u \in \mathfrak{P}^{m}$ and $d \in \mathfrak{X}^{n}$. Here, $d(x, t)$ may represent external diaturbasces as well as unmodeled dynamica : the key point in that it is unmessured. The general state and input transformation

$$
\begin{aligned}
& z=T(x) \\
& z=\alpha(x)+(I+\beta(x)) v
\end{aligned}
$$

transform (2) to one of several forms depending upon the selected technique.

We consider here a compariaon of the Global State Linearization [5] (GSL) technique and the Approximate Linearization (AL) developed by Krener and ca-workers [7], [9]. Using the following features as meacures, the AL represents a riguificanthy superior binearization approach:

- Transformed Coordinate System

- Disturbance Effects

- Involutivity Restrictions

- Optimization of Possible Tranaformation

These inves will now be discuned in more detail.

\subsection{Transformed Coordinate Syatem}

GSL traneforms systems of the form (2) into the following dynamical syotem:

$$
\dot{z}=\left(\begin{array}{ll}
0 & 1 \\
0 & 0
\end{array}\right) z+\left(\begin{array}{l}
0 \\
1
\end{array}\right) v+\left[\frac{\partial T}{\partial x} d(x, t)\right]_{s=T-1(s)}
$$

Although the resultant nominal state dynamics are linear, they are in Brunovsky canonical form in which many of the states bave lost their physical sifnificance. For process systern in which the states are typically temperatures and concentrations, the transformed variables may represent unmeasurable quantities or highly nonlinear functions of the measurable variables. Another problem with the so called "global" techniques is the fact that the state transformation $T(x)$ is only a local diffeomorphim and therefore these techniques can only be applied over finite regions of the phase space.

AL also handles systems of the form in (2), but for convenience we will represent the nominal systern as a series expansion of the terms in equation (2):

$$
\dot{z}=\begin{gathered}
F x+f^{(2)}(x)+\cdots+f^{(p)}(x)+ \\
\left(G+g^{(1)}(x)+\cdots+g^{(n-1)}(x)\right) w+\alpha^{(a+1)}(x, w)
\end{gathered}
$$

The following structure is imponed upon the state trassformation:

$$
z=x-\phi(x)(x)
$$

The resultant dynamical aystem is linear in the stale dynamics up through order $p$ terms:

$$
i=F z+G v+\left[\frac{\partial T}{\partial z} d(z, t)\right]_{s=T-1}(x)+O^{0+1}(z, v)
$$

This particular choice of state traneformation (7) and input (4) traneformation leads to first onder terms $F$ and $G$ which are identieal to the reapective term in the fint order approcimation of the original dynamical syotem. Technically, we say that the new vari ables $z$ bave pth-order contact with the original variables $x$. This i- 
contrasted with the GSL in which the transformed coordinates have only zero order contact with the oripinal variables (higher derivatives do not match). The implications for control design are obvious. We can calculate optimal linear controllers for the first order approximation of our original syatem and apply them directly to the AL system. For the GSL approach, one must first translate the desired first order dynamics in $x$ to the new coordinate system before the corresponding linear control law can be calculated.

\subsection{Disturbance Effects}

Another weakness of the GSL technique is revealed in the incorporation of disturbances and unmodeled dynamics into the transformed coordinates. It can be seen in equation (5) that even simple linear disturbances are transformed into potentially pathological operators in the linearized coordinate system. Simple perturbations in $d$ may dramatically effect the already critically stable nominal system (open bop). This is contrasted with the AL approach in which the transformation Jacobian matrix has zero-order terms equal to identity. The higher order terms are simple polynomials in $x$. In effect, this minimizes the "nonlinear" nature of the disturbances.

\subsection{Involutivity Restrictions}

A resriction on the nonlinear systems which admit GSL solutions is an involutivity condition, the computation of which becomes quite difficult for large order systems. Typical chemical engineering processes, particularly complex reaction systems, violate this constraint [8]. In contrast, the AL approach requires only that the system be "approximately involutive" or order- $\rho$ involutive [9]. This is a milder constraint in the that it allows an order $\rho$ remainder term whereas GSL requires zero remainder.

\subsection{Optimization of Possible Transformation} One of the real strengths of the AL approach lies in its flexibility to "optimize" the resultant solution. Consider the mapping from the transformations $(\phi, \alpha, \beta)$ to the functions $(f, g)$. For $\mathrm{AL}$, one can represent this mapping in terms of the polynomial coefficients of the various polynomial functions. (A straightforward interpretation is not possible for the GSL.) If this mapping has a non-trivial kernel, then a parametrized family of solutions results. The parameters are selected to minimize the "size" of $\alpha, \beta$ and $\phi$. If the magnitude of $\phi$ is minimized, then the mupping from $x$ to $z$ becomes closer to identity. Similarly, minimixing $\alpha$ and $\beta$ yields a mapping from $u$ to $v$ that is close to identity. Thus the linearization is accomplished with minimal nonlinear "distortion" of the original system.

Similarly, if the map is deficient in rank, then a linearization solution is not possible (i.e. the system is not p-order involutive). In this case, one can search over the space of solutions $(\tilde{f}, \tilde{g})$ which are linearizable. An optimization is done to minimize the distance between $(f, g)$ and $(\tilde{f}, \tilde{g})$. The reader is referred to [7] for the optimization algorithm and a discussion of the relevant metrics used to define the various sizes and distances.

In effect, the constraint in section 2.3 is removed. This will be demonstrated with a non-inwolutive example in the next section. This systematic procedure for finding the "closest" linearizable system reveals the flexibility of the AL approach.

\subsection{Summary}

It has been shown in equations (5) and (8) how the so-called linearization techniques actually result in nonlinear systems when the effects of disturbances and unmodeled dynamies are accounted for. This is represented in Figure 1 where the shaded blocks are nonlinear operators. At the center of this structure are the input transformation, nonlinear plant, and state transformation. It is clear to see the effect of unmodeled dynamics and disturbances on the stability of the open loop transformed system. $\Delta_{1}$ represents the difference between the true nonlinear dynamics and the assumed model. For instance, the Quadratic Approximate Linearization scheme (QAL), $\rho=2$, has a $\Delta_{1}$ term to represent order 3 and higher effects. $\Delta_{2}$ represents the nonlinear effect of the disturbance acting through the Jacobian of the transformation. Finally, $\Delta_{\mathbf{3}}$ represents the nonlinearity associated with the actual coordinate transformation itself and its effect upon the set-point signal.

The preceding four sections have clearly enumerated the incentives for using Approximate Linearization. This overall practicality

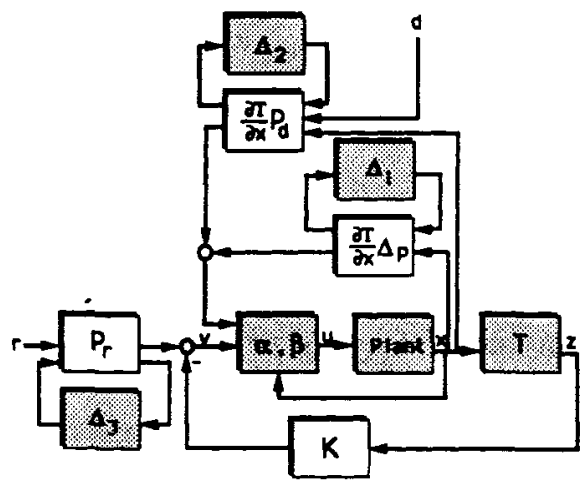

Figure 1: General Linearization Structure

motivates its use in the present design. In addition, there are extensions of this technique to systems in which the outputs are nonlinear functions of the states. Although they are not explored here, they represent an attractive framework for pursuing input-outpot linearization.

\subsection{Application to a Non-Involutive System}

Consider a continuous stirred tank reactor (CSTR) in which the following isothermal, liquid-phase chemical reactions take place :

$$
A \stackrel{1,2}{=} B \stackrel{3,4}{=} C
$$

The following rate expressions hold: $r_{1}=k_{1} C_{A}, r_{2}=k_{2} C_{B}^{2}, r_{3}=$ $k_{3} C_{B}^{2}$ and $r_{4}=k_{4} C_{C}$. Manipulation of the concentration of $\mathrm{C}$ (desired output) will be accomplished by the flow rate of a feedback stream consisting of primarily component $B$ which has been separated from the aqueous solution by a drying process. The following choice of physical parameters $\left(\mathcal{D a}_{1}=3.0, \mathrm{Da}_{2}=0.5, \mathrm{Da}_{3}=\right.$ 1.0, $\left.D a_{1}=2.0, \lambda_{1}=0.1, \lambda_{b}=0.75, \lambda_{c}=0.15\right)$ and operating point $\left(u_{f 0}=.5, x_{00}=0.33, x_{10}=0.79, x_{c 0}=0.21\right)$ leads to the normalized dimensionless mass balances:

$$
\begin{aligned}
& \dot{\tilde{x}}=\left(\begin{array}{ccc}
-3.95 & .790 & 0.0 \\
3.0 & 2.994 & 2.0 \\
0.0 & 1.580 & -2.925
\end{array}\right) \tilde{x}+\left(\begin{array}{l}
.033 \\
.592 \\
.032
\end{array}\right) \tilde{u} \\
& +\left(\begin{array}{c}
.5 \tilde{x}_{b}^{2} \\
-1.5 \tilde{x}_{b}^{2} \\
1.0 \tilde{x}_{b}^{2}
\end{array}\right)+\left(\begin{array}{ccc}
0.1 & 0.0 & 0.0 \\
0.0 & 0.75 & 0.0 \\
0.0 & 0.0 & 0.15
\end{array}\right)\left(\begin{array}{l}
\tilde{x}_{c} \\
\tilde{x}_{b} \\
\tilde{x}_{c}
\end{array}\right) \tilde{u}
\end{aligned}
$$

Note that this system is second order and is in control-linear form. However, it is straightforward to show that this system is not involutive. Using the MATLAB software for QAL (see Acknowledgements) one that the following plant is in fact involutive:

$$
\begin{aligned}
\dot{\tilde{x}} & =\left(\begin{array}{ccc}
-3.95 & .790 & 0.0 \\
3.0 & 2.994 & 2.0 \\
0.0 & 1.580 & -2.925
\end{array}\right) \tilde{x}+\left(\begin{array}{l}
.033 \\
.592 \\
.032
\end{array}\right) \bar{u} \\
& +\left(\begin{array}{ccc}
-.00058 \tilde{x}_{a} \tilde{x}_{b} & .490 \tilde{x}_{b}^{2} & -.00056 \tilde{x}_{b} \tilde{x}_{c} \\
& -1.5 \tilde{x}_{b}^{2} & \\
.00027 \tilde{x}_{a} \tilde{x}_{b} & 1.005 \tilde{x}_{b}^{2} & .00026 \tilde{x}_{b} \tilde{x}_{c}
\end{array}\right) \\
& +\left(\begin{array}{ccc}
0.112 & -0.012 & 0.027 \\
-.00039 & 0.750 & -.00086 \\
-.0052 & 0.011 & 0.138
\end{array}\right)\left(\begin{array}{l}
\tilde{x}_{a} \\
\tilde{x}_{b} \\
\tilde{x}_{c}
\end{array}\right) \tilde{u}
\end{aligned}
$$

The remarkable closeness of the two systems is attributable to a remainder term which satisfies the approximate involutivity condition for syptem (11). Simulation results confirm that when the QAL for (11) is applied to (10), the resultant closed boop exhibits lese nonlinear behavior than the original nonlinear plant with simple linear control [2] 


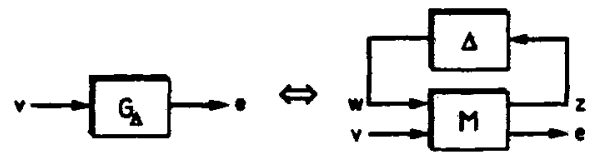

Figure 2: General Framework for SSV Analyais

\section{Structured Singular Value Concepts}

3.1 General Framework

We consider the system shown in Figure 2 where $\mathbf{M}(\mathrm{s})$ is a linear time invariant operator and $\Delta$ is a nonlinear operator with the following block structure:

$$
\Delta \triangleq\left\{\operatorname{diag}\left[\delta_{1} I_{r_{1}}, \ldots, \delta_{m} I_{r_{-}}, \Delta_{1}, \ldots, \Delta_{n}\right]\right\}
$$

This structure can be arrived at from any interconnection of linear blocks and nonlinear perturbations, $\Delta$. In the diagram, the input $v$ represents set points, disturbances and noise, the output $e$ represents error signals. In this framewort, the control analysis problem focuses on two key questions : first, is the system stable for all perturbations in some prescribed set (robust stability); and second, does the error remain in a desired bounded set for all perturbations and inputs in some appropriate sets (robust performance).

Practically speaking, this approsech will be applied to plants with input signals of bounded energy or bounded power, and the results guarantee that the outputs are also bounded in energy or power, respectively. For the class of bounded energy signals, mild smoothness conditions guarantee that the signal goes to rero asymptotically. It should be noted that the clase of input ignals can be broadened by the inclusion of weights which are incorporated into the nominal plant M. This can be used, for example, to inctude stepe in the in put elos. Similarly, the output aignals can be weighted to incorporate performance criteria.

\subsection{Stability and Performance Results}

In this section, the results of [3] are brieffy summarized. Consides again Figure 2 and an appropriate partitioning of $\mathbf{M}$ :

$$
\left(\begin{array}{l}
e \\
z
\end{array}\right)=\left(\begin{array}{ll}
M_{11} & M_{12} \\
M_{21} & M_{22}
\end{array}\right)\left(\begin{array}{l}
v \\
v
\end{array}\right)
$$

Conpecting the loop between $z$ and $w$ yields the linear fractional transformation (LFT) representation for the overall operator $G_{\Delta}$ :

$$
e=F_{l}(M, \Delta)_{v} \triangleq\left(M_{11}+M_{12} \Delta\left(I-M_{20} \Delta\right)^{-1} M_{21}\right] v
$$

Since it is required that the nonlinearities are conic sector bounded, it is without bose of generality that $\Delta$ in restricted to the class of bounded operators:

$$
B \Delta:=\{\Delta \in \Delta \mid \Delta \in \operatorname{Cone}(0, I, I)\}
$$

We forgo formally defining a cone until the next section, it suffices to say that the operators in this class have well-behaved bounded properties. The small gain theorem can be used to arrive at sufficient conditions for robust stability and performance (RS) and (RP):

$$
\begin{array}{lll}
R S: & \left\|M_{22}\right\|_{\infty}=\beta_{R S} & \left(\beta_{R S}<1\right) \\
R P: & \left\|F_{\ell}(M, \Delta)\right\|_{\infty}=\beta_{R P} & \left(\beta_{R P}<1\right) \text { for all } \Delta \in B \Delta
\end{array}
$$

These conservative conditions can be improved by the introduction of constant scaling matrices which commute with the perturbation block $\Delta$. In this context, commutivity is defined to be:

$$
\left\|D \Delta D^{-1}\right\|_{\infty} \leq\|\Delta\|_{\infty}
$$

For the uncertainty structure given in equation (12), one appropriately structured commuting set is:

$$
\begin{aligned}
& D \triangleq\left\{\operatorname{diag}\left[D_{1}, \ldots, D_{i m}, d_{1} I_{k_{1}}, \ldots, d_{n} I_{k_{m}}\right]\right. \\
& \mid D_{i} \in C^{r_{i} \times r_{i}} \text { is invertible, } d_{i} \neq 0
\end{aligned}
$$

Incorporating these sealing mutricen, we can arrive at lewe conservative conditions for Robest Stubility and Robent Peformunce:

$$
\begin{aligned}
& R S^{\prime}:\left\|D M_{23} D^{-1}\right\|_{\infty}=\beta_{R S^{\prime}}\left(\beta_{R S^{\prime}}<1\right) \\
& R P^{\prime}:\left\|F_{l}\left(\left[\left(\begin{array}{cc}
I & 0 \\
0 & D
\end{array}\right) M\left(\begin{array}{cc}
I & 0 \\
0 & D^{-1}
\end{array}\right)\right], \Delta\right)\right\|_{\infty}=\beta_{R P^{\prime}}
\end{aligned}
$$

In [3], the motivation was presented for carrying out the SSV calevlation in the time domain because of the compatational attractivenew of the resulting calculations. Consider now the discrete map $N(z)$ which is calculated from $M(s)$ with the norm-preserving bilinear tranaformation : = $\frac{1-2}{1+x}$ mapping the unit disk to the right balf plane. The map is appropriately partitioned :

$$
\left(\begin{array}{c}
x_{k+1} \\
e_{k} \\
z_{k}
\end{array}\right)=\left(\begin{array}{lll}
N_{11} & N_{12} & N_{13} \\
N_{21} & N_{32} & N_{23} \\
N_{31} & N_{33} & N_{33}
\end{array}\right)\left(\begin{array}{c}
x_{k} \\
v_{k} \\
v_{k}
\end{array}\right)
$$

where $w_{k}=\Delta\left(k, z_{k}\right)$ and for each $k, \Delta$ is an element of the prescribed uncertainty set $B \Delta$. Now a coordinate transformation, $\hat{T}$, in introduced a scaling on the state variables. In an analogons manner to the commuting $D$ scales, the coordinate transformation reduces the conservation of the time domain result.

Theorem 1 (Robust Performance) Given a system $N$ and block structure $\Delta$. Suppose $\Delta$ is inside Cone $(0, I, I)$. If there ere approprintely pertitioned constant acating matrices $T$ and $D$ onch thet:

$$
\bar{\sigma}\left(\left(\begin{array}{ccc}
\hat{T} & 0 & 0 \\
0 & I & 0 \\
0 & 0 & D
\end{array}\right) N\left(\begin{array}{ccc}
\hat{T}^{-1} & 0 & 0 \\
0 & I & 0 \\
0 & 0 & D^{-1}
\end{array}\right)\right)=\beta<1
$$

then the wacertain aystem:

$$
\begin{gathered}
\left(\begin{array}{c}
x_{k+1} \\
e_{k} \\
x_{k}
\end{array}\right)=\left(\begin{array}{lll}
N_{11} & N_{12} & N_{13} \\
N_{21} & N_{22} & N_{23} \\
N_{31} & N_{32} & N_{30}
\end{array}\right)\left(\begin{array}{c}
x_{k} \\
v_{k} \\
v_{k}
\end{array}\right) \\
w_{k}=\Delta\left(k, z_{k}\right)
\end{gathered}
$$

is zero-inpud exponentially steble and if $x_{0}=0$ and $\left\{v_{k}\right\}_{k=0}^{\infty} \in \ell_{2}$, then $\left\|e k_{2} \leq \theta\right\| v \|_{k_{2}}$.

Proof See [S].

\subsection{State Bounded Result}

For this atudy, it is neceseary to impone bounds on the atate variables to guarantee the invertibility of the linearizing tranformations a well as to facilitate the calculation of conic sector bounds. The region will consist of a scaled unit hypersphere which becomes a hyperellipeoid in the original variables.

The SSV yields performance results with the norm of an error signal being bounded by a norm on the input signal. If we select as an error signal $x_{2}$, the sealed states, then we can calculate an upper bound for $x$, as a function of the inputs and the initial conditions. This particular value of the SSV will be denoted $\boldsymbol{\beta}_{B S}$ :

$$
\text { Theorem } 1 \text { and }\left\{v_{k}\right\}_{t=0}^{\infty} \in l_{2} \Rightarrow\left\|x_{1}\right\|_{l_{2}}^{2} \leq \beta_{B}^{2}\|v\|_{l_{2}}^{2}+\left\|\tilde{x}_{0}\right\|_{l_{2}}^{Z_{2}}
$$

Here $\tilde{x}_{0}=\hat{T} x_{0}$ represents the transformed states of the cloeed loop syatem In order to consider only the original states of plant, a correction must be made for the transformation $\hat{T}$. If the initial controllet states are chooen to be zero, then the tradeof between the efiect of initial conditions and input magnitude is given by the ellipee:

$$
\left\|x_{s}\right\|_{\ell_{2}}^{2} \leq \beta_{B s}^{2}\|\nabla\|_{\ell_{2}}^{2}+\tilde{\sigma}^{2}(\hat{T})\left\|x_{s},\right\|_{R_{2}}^{2_{2}} \leq 1
$$

The spatial result follows directly from this temporal norm boand and the fact that at a given $k,\left\|x_{o},\right\|^{2} \leq \sum_{k=1}^{\infty}\left\|x_{s k}\right\|^{2}=\left\|x_{,}\right\|_{R_{2}}$. 


\section{Conic Sector Bounded Nonlinearities}

\subsection{General Description}

In this section, the class of nonlinear operators which are to be considered is formally defined. It is required that the operator $y=$ $N(x)$ be inside a conic sector or cone as defined by:

$$
\text { Cone }(\mathrm{C}, \mathrm{R}, \mathrm{S}) \triangleq\{(x, y) \mid y=C x+S \Delta(R x),\|\Delta(x)\| \leq\|x\|\}
$$

For $y \in, x \in$ and a square $\Delta$ structure of size $d$, then $S \in$ $\Re^{p \times d}, R \in \Re^{d \times n}$ and $C \in \Re^{\times \times n}$. A simpler definition of a cone is given for the case where $S=I$ :

$$
\operatorname{Cone}(\mathrm{C}, \mathrm{R}, \mathrm{I}) \triangleq\{(x, y)\|y-C x\| \leq\|R x\|\}
$$

In simplest terms, the cone center $C$ represents the best linear approximation of the nonlinear operator over the range of interest. The radii $R$ and $S$ give some measure of the error associated with this representation. A key point to note is that the Cone(C,R,S) contains many different operators, some of which may be considerably more pathological than the original nonlinear operator.

In terms of LFTs, the conic sector has a convenient representation which lends itself naturally to SSV analysis:

$$
\operatorname{Cone}(\mathrm{C}, \mathbf{R}, \mathbf{S})=F_{\mathrm{u}}\left(M_{c}, \hat{\Delta}\right) \text { where } M_{c}=\left(\begin{array}{ll}
0 & R \\
S & C
\end{array}\right)
$$

and $\hat{\Delta}$ is inside the Cone $(0, I, I)$. This shows quite clearly how the nominal plant $C x$ is perturbed via the terms $R$ and $S$. A minimization of these two factors yields the least "uncertain" or most linear system. The term $R$ accounts for the interactions between the inputs to the operator and the term $S$ takes into account the coupling between the outputs of the system. For example, a perfectly diagonal map such as $y_{i}=f_{i}(x)$ for $i=1, p$ can be described by a cone with a diagonal $R$ term and a diagonal $\Delta$ structure with $p$ uncertain gains (the diagonal $S$ term can be abeorbed through the $\Delta$ block into the $R$ term).

\subsection{Optimal Cones}

The objective of a minimally conservative paradigm is to reduce the effect of the $S \Delta R$ term in the conic sector description of our nonlinear plant. This is determined by two factors:

- The overall magnitude of $R$ and $S$

- The complexity of the $\Delta$ structure

The balancing of these two factors to yield the least conservative SSV calculation is a formidable task, involving an elaborate iterative scheme. A simple algorithm is proposed here to arrive at a suboptimal solution to this problem (though not necessarily the global optimum).

In general, the maximum (sensible) $\Delta$ is a diagonal matrix containing $n p$ independent gains. This assumes completely independent gains for each input-output matching in the mapping of $N$. At the other extreme, the simplest nonlinear operator could be described with a single uncertain gain. This is true in the conic sector representation for the dynamics of a simple CSTR [3]. Simpler $\Delta$ struetures are more aftractive from a computational perspective, and in fact the SSV is equal to its computable upper bound for some simple structures in the case where $\Delta$ is linear (but possibly time-varying) [11].

For a fixed $\Delta$ structure, a simple geometric argument will be made for the definition of a minimally conservative cone. Consider the case of a scalar nonlinear operator $(S=1)$. In the table below is shown the geometric interpretation of various conic sectors. In order to minimize the region inside the cone, one can minimize the hyperdimensional angle (or sum of such quantities) which define the region. It can be shown that this is equivalent to a minimization of the Frobenius norm of the matrix $R$. This result can also be derived from the fact that the Frobenius norm is an upper bound for the infinity norm. Using the conic sector definition (22) we get:

$$
\frac{\|S \Delta(R x)\|}{\|x\|^{2}} \leq \frac{\|S\|\|\Delta(R x)\|}{\|x\|^{2}} \leq \frac{\|S\|\|R x\|}{\|x\|^{2}} \leq\|S\|_{F}\|R\|_{F}
$$

\begin{tabular}{|c|c|c|}
\hline \multicolumn{3}{|c|}{ Conic Sector Regions } \\
\hline d & $\mathbf{n}$ & Region \\
\hline 1 & 1 & 2 Flat Slices \\
\hline 1 & 2 & 2 Infinitely Deep Slices \\
\hline 2 & 2 & 2 Square Based Pyramids \\
\hline
\end{tabular}

Table 1

Three possibly distinct solutions to the conic sector minimization problem can be envisioned corresponding to three $\Delta$ structures. The Feasible solution minimizes the Frobenius norm of the smallest $\Delta$ structure which can envelop the nonlinear operator. The Fvll solution minimizes the Frobenius norm of the largest sensible $\Delta$ strueture ( $n p$ independent gains). As the size of the $\Delta$ structure increases, the Frobenius norm must necessarily decrease (i.e. the smaller Feasible structures are all subsets of a larger structure). As these are the limiting cases, the Global Optimal solution, which minimizes the upper bound on the SSV must lie between them. The tradeoff between simple $\Delta$ structure and small Frobenius norm is balanced at this point.

\subsection{Numerical Calculations}

A nonlinear program (NLP) is set up to calculate the solution which minimizes the Frobenius norm for a given $\Delta$ structure. The program requires $q$ data points consisting of an input vector $x$ and corresponding output vector $y$. As most of the nonlinearities for this study are monotonic and constrained to a finite region in $x$, it was often sufficient to calculate the data points along the boundary of the hyper-ellipeoid. The general sebeme of the the NLP is to minimize the Frobenjus norm with the constraint that the data lie inside the calculated conic sector. Obviously, the larger the value of $q$, the more accurate the calculated conic sector and the greater the computational complexity of the NLP. For the case where the d sub-blocks of $\Delta$ are scalar, the NLP can be written:

$$
\begin{gathered}
\text { Min } \eta \\
\left(\sqrt{\sum_{i=1}^{i} \sum_{j=1}^{d} S_{i j}^{2}}\right)\left(\sqrt{\sum_{i=1}^{d} \sum_{j=1}^{n} R_{i j}^{2}}\right)=\eta \\
k=1, q\left\{\begin{array}{cc}
\sum_{i=1}^{n} C_{1 i} x_{i k}+\sum_{i=1}^{d}\left[S_{1 i}\left(\sum_{j=1}^{n} R_{i j} x_{j k}\right) \delta_{i k}\right] & =y_{1 k} \\
\vdots & \\
\sum_{i=1}^{n} C_{p i} x_{i k}+\sum_{i=1}^{d}\left[S_{p i}\left(\sum_{j=1}^{n} R_{i j} x_{j k}\right) \delta_{i k}\right] & =y_{k k} \\
\left|\delta_{1 k}\right| & \leq 1 \\
\vdots & \leq 1
\end{array}\right.
\end{gathered}
$$

where $\left\{\left(y_{1 i}, \ldots, y_{p i}\right),\left(x_{1 i}, \ldots, x_{n i}\right)\right\}$ are the given data points $(i=$ $1, q)$. The total number of variables is $(1+d n+d p+p n+d q)$ and the total number of constraints is $(1+p q+d q)$. It is important to note that the above algorithm can be used to find the linear bounds on an arbitrary polynomial approximation (cone center) to the nonlinear function. The additional variables introduced appear linearly in the constraint equations (i.e. $y=C_{1} x+C_{2} x^{2}+\cdots$ ). This approach will be used in conjunction with the QAL scheme.

\section{Application to a Nonlinear CSTR}

\subsection{CSTR Model}

The mass and energy balances for a CSTR with first order, irreversible, exothermic kinetics $(A \rightarrow B)$ are give by :

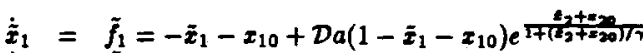

$$
\begin{aligned}
& \dot{\tilde{x}}_{2}=\tilde{f}_{2}-\beta u+d=-\tilde{x}_{2}-x_{20}-\beta\left(\tilde{u}+u_{0}\right)+d
\end{aligned}
$$

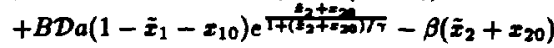

(using the dimensionlese quantities defined in the nomenclature). This simple model has two state variables (reactant concentration, reactor temperature). The control problem focuses on the SISO regulation of the reactor temperature by manipulating the cooling water temperature while subjected to disturbances in the feed stream temperature. The values of the dimensionless parameters 
are [1]: $D_{a}=0.072, B=8, \beta=0.3, \gamma=20$ and $x_{c}=0.0$. These conditions lead to multiple steady states and operation is chooen at the unstable point : $\left(u_{0}=-0.20, x_{10}=0.5, x_{20}=3.03\right)$.

5.2 Linearizing Transformations and Uncertainty Description

We consider three controller synthesis techniques (GSL, QAL and simple linesr (P, PI)) as well $\approx 2$ diaturbance classes (bounded energy and steps). The results are extensively documented in [2]. The GSL results have been publiohed ebewhere [1],[4], we merely summarize here. The state and input transformations:

$$
\begin{array}{rll}
z_{1} & =T_{1}(x)= & x_{1}-z_{10} \\
z_{2} & =T_{2}(x)= & \tilde{f}_{1}\left(x_{1}-x_{10}\right) \\
\alpha(x) & =\left\langle d T_{3}, f\right\rangle \\
\beta(x) & =\left\langle d T_{2}, g\right\rangle-I
\end{array}
$$

yield, in transformed coordinates, the dynamical system:

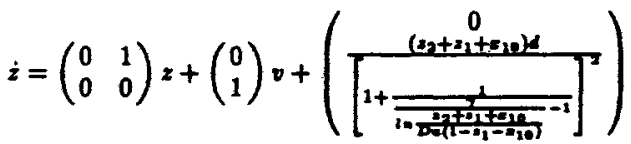

It is clear to see the constraints impoeed by inverting $T_{2}(x)$ in equar tion (27). For general problems, this inherent fimitation restriets the application of state linearization techniques to local regions in the phase space. For the purpose of this study, we consider the region of interest to be the interior of the ellipse:

$$
\frac{z_{1}^{2}}{.08^{2}}+\frac{z_{2}^{2}}{.55^{2}}=1
$$

for QAL. This corresponds roughly to a circle of radius 0.1 in the $z$ domain for GSL.

For the QAL, it is necessary to calculate nominal quadratic plant. Using the NLP described in the previous section, it is found that the second order plant with the tightest conic sector over the region of interest (equation (29)) is given by:

$$
\begin{aligned}
& \bar{x}_{1}=-2 \tilde{x}_{1}+.381 \tilde{x}_{2}+.020 \tilde{x}_{1}^{2}-.761 \tilde{x}_{1} \bar{x}_{2}+.127 \tilde{x}_{2}^{2} \\
& \tilde{x}_{2}=-8 \bar{x}_{1}+1.75 \bar{x}_{2}+.320 \bar{x}_{1}^{2}-6.09 \tilde{x}_{1} \bar{x}_{2}+1.02 \tilde{x}_{2}^{2}+0.3 \tilde{u}
\end{aligned}
$$

The uncertainty i characterized by a single uncertain gain with radii: $S=\left(\begin{array}{ll}1 & 8\end{array}\right)^{T}, R=\left(\begin{array}{ll}0 & 0.0067\end{array}\right)$. This can be compared with the tightest conic sector aseociated with a first order approximation of the nonlinear plant:

$$
\dot{\bar{x}}=\left(\begin{array}{ll}
-2 & 0.377 \\
-8 & 1.718
\end{array}\right) \tilde{x}+\left(\begin{array}{c}
1 \\
0.3
\end{array}\right) \tilde{z}
$$

and its asociated uncertainty radii: $S=\left(\begin{array}{ll}1 & 8\end{array}\right)^{T}, R=\left(\begin{array}{ll}0 & 0.104\end{array}\right)$. Using the objective in our NLP as a meesure, the quadratic approximation reduces the nonlinear uncertainty by a factor of 15 . The second order system in equation (30) yields an exact quadratic biearization with the following state and input transformations :

$$
\begin{aligned}
z_{1}=T_{1}(x) & =\tilde{x}_{1}-0.7388 \tilde{x}_{1}^{2} \\
z_{2}=T_{2}(x) & =\tilde{x}_{2}+3.9320 \tilde{x}_{1}^{2}-3.4751 \tilde{x}_{1} \tilde{x}_{2}+0.3333 \tilde{x}_{2}^{2} \\
\alpha(x) & =-1.3304 \bar{x}_{1}^{2}-4.9235 \tilde{x}_{1} \tilde{x}_{2}+0.9311 \tilde{x}_{2}^{2} \\
\beta(x) & =-3.4751 \bar{x}_{1}+0.6667 \tilde{x}_{2}
\end{aligned}
$$

In the transformed coordinates we have the same linear approximation:

$$
\dot{z}=\left(\begin{array}{ll}
-2 & 0.3808 \\
-8 & 1.7467
\end{array}\right) z+\left(\begin{array}{c}
0 \\
0.3
\end{array}\right) v+\left[\frac{\partial T}{\partial x}\left(\begin{array}{l}
0 \\
d
\end{array}\right)\right]_{x=T^{-1}(z)}+\Delta P
$$

where the Jacobian is calculated from equation (32). The quadratic transformation is also invertible over the region defined in equation (29). The other term in this equation, $\Delta P$, accounts for both the error in approximating the nonlinear plant by a second order system and also for the order 3 and higher terms inherent in the QAL.

In summary for the disturbance problem, the GSL scheme requires a single uncertainty block to represent the nonlinear effect of the disturbance. Thi block is optimally modeled with two uncertain nonlinear gains. The QAL technique requires an uncertainty block for both the nonlinear dieturbance effect and the plant modeling error. In this case, the optimal uncertainty description has three nonlinear gains. Finally, the simple linear control approach requires a single uncertain gain to represent the error inherent in the first order approximation. The strueture of the various uncertainty blocts and the magnitude of their radii are listed in the table belom. It should be pointed out that concluaions sbout the various schemes besed on these date alone should be drawn carefully. The schemes represent rather diferent coordinate systems a well aneertainty structures.

\begin{tabular}{|c|c|c|}
\hline \multicolumn{3}{|c|}{ Uncertainty Descriptions } \\
\hline \hline Method & $\Delta$ structure & $\|S\| F\|F\|_{F}$ \\
\hline GSL & $\left(\begin{array}{ll}\delta_{1} & 0 \\
0 & \delta_{2}\end{array}\right)$ & 0.20 \\
\hline QAL & $\left(\begin{array}{ccc}\delta_{1} & 0 & 0 \\
0 & \delta_{2} & 0 \\
0 & 0 & \delta_{3}\end{array}\right)$ & 0.61 \\
\hline Linear & $\left(\delta_{1}\right)$ & 1.10 \\
\hline
\end{tabular}

Table 2

\subsection{Analysis of Stability Properties}

We restrict our attention in this paper to a particnlar performance criterion - namely the bounding of the states while the plant in subjected to two claseses of disturbances. In the first ease, we consider bounded inputs of bounded energy (e.g. decaying sinusoids). In the eecond case, we consider the clane of inputs of bounded energy pased through the filter $\frac{1}{3}$ (e.g. steps).

\subsubsection{Bounded Energo Diaturbances}

In this case it is sufficient to consider simple proportional control in the outer loop of Figure 1. No integral action is required for feed temperature diaturbances of bounded energy to guarantee zero offset. The controller gains $[1.35,2.12]$ as found in [1] are used foe the GSL, and the gains $[-16.9,6.23]$ yield the same clowed loop polen for the QAL (and the simple linear appronch). To simplify our comparion, we allom all three system to have the same cloced bop dynamics in the treasformed variables. A more realistic comparieon might involve calculating a controller to give the same dynamic in the original variables but as we have seen (sec. 2.1) this is not eaily done for the GSL.

The analysis procedure involves varying the magnitude of $d$ and calculatiog a corresponding value of $\beta_{B S}$. Then we can use equation (21) to calculate a bound on the initial state. For the general robust performance problem, one can use this approach to calculate the tradeof between initial condition and input magnitude of feets on the computed performance guarantee. For the state bound problem, the tradeof curves are plotted in Figure 3. Recall that this approach yields sufficient results, so the curves in thi diagram represent a lomer bound on the tolerable disturbance and initial condition magnitudes which give riee to bounded states. An additional point is given in the diagram to show an actual simulation in which the states escupe the bound in (29). This represents an upper bound on the tolerable disturbance magnitude (zero initial condition) and the proximity of the points along the $y$-axis shoms the promise of this technique.

In Figure 4, we depiet the varions state bounds $\boldsymbol{a}$ they are mapped into the true $x_{1}-x_{2}$ coordinate system. As expected, the QAL contour shows minimal deformation of the original ellipare a compared to the GSL contour. It is evident from this figure that a precise comparison of the results is not poesible. Each result guarantees stability over a different region in the phase space. Included in this diagram is the phase portrait of the response of the simple linear aystem to the input dieturbance $0.3 e^{-.25 t} \sin (.5 t)$. The response of the QAL and GSL are virtually identical to the depicted response for this input. This indicates that Table 2 is a ressonable measuse of the conservatism inberent in analyzing the three approaches in the SSV framework.

5.3.2 Step Input.

The above procedure is repeated for persistent step disturbances with PI control in the outer loop for aysymplotic tracking. The 


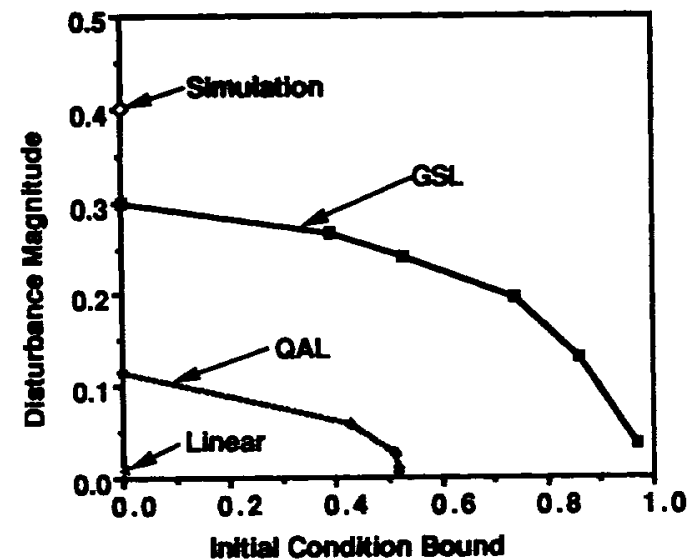

Figure 3: Bounds for which closed loop stability is guaranteed (Bounded $\mathcal{L}_{2}$ Signals)

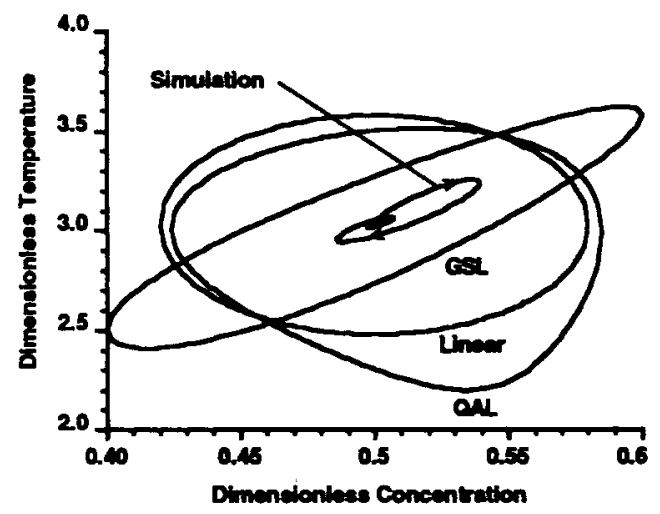

Figure 4: Bounds on State Trajectories

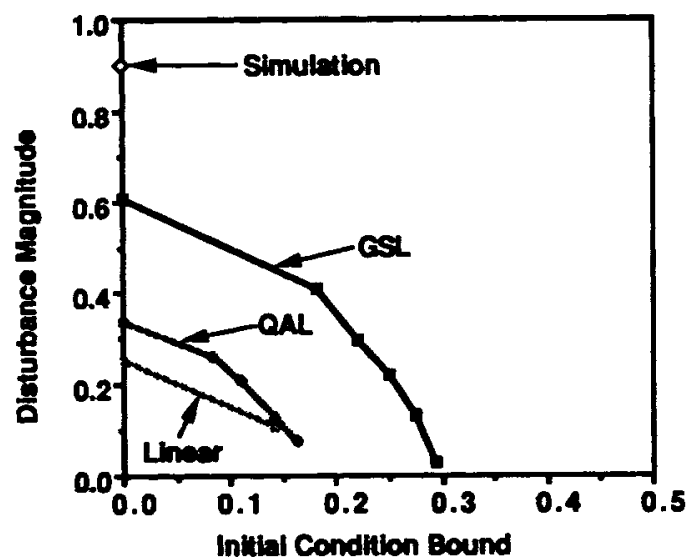

Figure 5: Bounds for which closed loop stability is guaranteed (Step-like Signals)

controller gains are $[4.93,3.93]$ for the GSL and the error in $z_{1}$ is integrated with $\tau_{I}=0.422$. For the QAL (and linear) schemes, the gains which yield the same closed loop poles are $[-17.27,12.25]$ and $\tau_{I}=0.0482$. The GSL approach suffers from the meakness that only $z_{1}$ represents a physically meaningful quantity to integrate. This is opposed to the flexibility inherent in the QAL scheme to integrate a reasonable estimate of $x_{2}$. The tradeoff between disturbence bounds and initial condition magnitudes is shown in Figure 5.

\section{Conclusion}

In this paper we have revealed the theoretical concepts which demonstrate that the Quadratic Approximate Linearization is a more practical linearization scheme than the Global State Linearization. The presence of external disturbances and unmodeled dynamics is manifested in relatively minimal nonlinear behavior in the QAL scheme. In addition, the broad applicability of this technique and potential for systematic optimization of the resultant solution further motivate its use. Coupled with extensions of the SSV to nonlinear systems, a control design procedure is developed which enables the analysis of stability and performance properties for these linearization schemes.

\section{Acknowledgements}

The authors wish to thank Sinan Karahan for providing us with a preliminary version of the MATLAB software for Approximate Linearization.

\section{References}

[1] Calvet, J.P., "A Differential Geometric Approach for the Nominal and Robust Control of Nonlinear Chemical Processes", Ph.D. Thesis, Georgia Institute of Technology, Atlanta, GA, 1989.

[2] Doyle III, F. J. and Morari, M., "Nonlinear Controller Design and Analysis for Uncertain Process Systems", in preparation, 1990.

[3] Doyle III, F. J., A. P. Packard and M. Morari, "Robust Controller Design for a Nonlinear CSTR", Chem. Eng. Sci., 44, 1929-1947, 1989.

[4] Hoo, K. A. and Kantor, J.C., "An Exothermic Continuous Stirred Tank Reactor is Feedback Equivalent to a Linear System", Chem. Eng. Cormm., 37, 1-10, 1985.

[5] Hunt, L.R., Su, R., and Meyer, G., "Global Transformations of Nonlinear Systems", IEEE Trans. Aut. Control, 24, 24-31, 1983.

[6] Isidori, A., Nonlinear Control Systems, Springer-Verlag, New York, NY, 1989

[7] Karahan, S., "Higher Degree Approximations to Nonlinear Systems", Ph.D., University of California, Davis, CA, 1988.

[8] Kravaris, C. and Kantor, J.C., "Geometric Methods for Nonlinear Process Control: A Tutorial", I. \& E.C. Res., in press, 1990.

[9] Krener, A.J., "Approximate Linearization by State Feedback and Coordin ate Change" ${ }^{n}$, Syst. Contr. Lett, 5, 181-185, 1984.

[10] Packard, A., "What's New with $\mu$ : Structured Uncertainty in Multivariable Contro", Ph.D. Thesis, University of California, Berkeley, CA, 1987.

[11] Packard, A. and Doyle, J., "Structured Singular Value with Repeated Scalar Blocks”, Proc. 1988 Am. Contr. Conf., 12131218.

\section{Nomenclature}

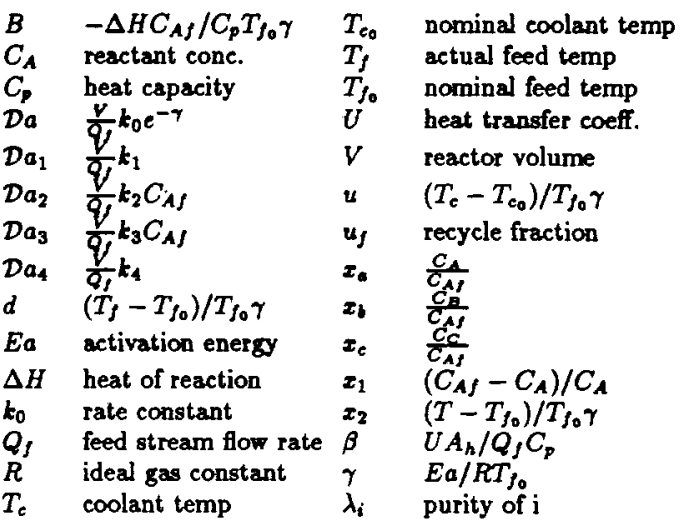

